\title{
Design and start-to-end simulation of an X-band RF driven hard X-ray FEL with LCLS injector
}

\author{
Yipeng Sun \\ SLAC National Accelerator Laboratory, Menlo Park, California 94025, USA
}

August 20, 2012

\begin{abstract}
In this note, it is briefly discussed the accelerator design and start-to-end 3D macro particles simulation (using ELEGANT and GENESIS) of an X-band RF driven hard X-ray FEL with LCLS injector. A preliminary design and LiTrack 1D simulation studies were presented before in an older publication [1]. In numerical simulations this X-band RF driven hard X-ray FEL achieves/exceeds LCLS-like performance in a much shorter overall length of $350 \mathrm{~m}$, compared with $1200 \mathrm{~m}$ in the LCLS case. One key feature of this design is that it may achieve a higher final beam current of $5 \mathrm{kA}$ plus a uniform energy profile, mainly due to the employment of stronger longitudinal wake fields in the last X-band RF linac [2].
\end{abstract}

\section{Overview and Accelerator design}

LCLS is the world's first hard X-ray FEL in routine operation, providing soft and hard X-rays to its users with good spatial coherence at wavelengths from $2.2 \mathrm{~nm}$ to $0.12 \mathrm{~nm}$ by varying the bunch energy from $3.5 \mathrm{GeV}$ to $15 \mathrm{GeV}[3]$.

Design and beam dynamic studies are performed for several X-band RF driven hard X-ray FEL drivers [4]. A low-charge hard X-ray FEL design [5] employs all X-band photoinjector and RF linac, which is optimized for a very low bunch charge of $10 \mathrm{pC}$ and an average SASE FEL power over 10 $\mathrm{GW}$ in a pulse length of $5 \mathrm{fs}$ (from start-to-end simulation) (in a short undulator length of $20 \mathrm{~m}$ ). Thanks to the small 3D emittance associated with the low bunch charge (short initial bunch length from photoinjector etc.), a linear bunch compression is achieved in two stages of bunch compressors without the assistance of harmonic RF linearization.

A second hard X-ray FEL design also features all X-band photoinjector and RF linac, but is dedicated for a normal bunch charge of $250 \mathrm{pC}$ [6]. The longitudinal phase space linearization is done in its first stage bunch compression with a specially designed bunch compressor which is composed of dipole, quadrupole and sextupole magnets. From start-to-end simulation using computer codes ASTRA [7], ELEGANT [8] and GENESIS [9], it is shown that this FEL achieves an average SASE FEL power over $30 \mathrm{GW}$ in a pulse length of $50 \mathrm{fs}$. 
Both the above two hard X-ray FEL design achieve a shorter SASE FEL saturation length, due to a shorter undulator period and a weaker undulator field. It is also noted that the low-charge hard X-ray FEL design [5] achieves a better final beam quality than the LCLS case.

Analytical and numerical simulation studies show that the tolerance (charge and timing jitter, transverse misalignment) is acceptable for both above two hard X-ray FEL design [5] [6].

Sketch of this compact $(<300 \mathrm{~m})$ hard X-ray FEL driver using X-band accelerators plus LCLS injector is shown in Figure 1 below. As mentioned above it employs LCLS injector, which means that to the point of BC1 end it is exactly the same as LCLS. The only difference is that the S-band $\mathrm{RF}$ in linac2 and linac3 is replaced by X-band RF. BC2 design is similar as LCLS, at a beam energy of $4.3 \mathrm{GeV}$. For a bunch charge of $250 \mathrm{pC}$, the initial RMS bunch length is $690 \mu \mathrm{m}$ from the S-band photoinjector. It is compressed to a final RMS length of either $6 \mu \mathrm{m}$ (3 kA peak current) or $4 \mu \mathrm{m}$ (5 kA peak current).

\section{Optics design}

The optics design of the linac, the bunch compressors and the matching sections are done in MAD8 [10] and then converted to ELEGANT [8]. The TWISS parameters of bunch compressor one (BC1) and two (BC2) are shown in Figure 2 below, which are similar with LCLS BC1 and BC2 (transverse phase space matching to minimize CSR induced transverse emittance growth). The start-to-end TWISS parameters of the overall accelerator are shown in Figure 3. An average beta function of 10 meters and 20 meters are employed in Linac2X and Linac3X, respectively, to minimize both dispersive and wake-fields-induced transverse emittance dilutions.

\section{ELEGANT simulation}

The beam dynamics simulation in the photoinjector is performed in ASTRA [7], and then a dumped beam is taken as the initial beam in ELEGANT simulation. First, second and third order optics effects are included in ELEGANT simulation, as well as all the collective effects, such as space charge effect, incoherent and coherent synchrotron radiation, transverse and longitudinal wake fields.

The RMS bunch length and transverse emittance evolution along the accelerator are shown in Figure 4, with the comments listed in its caption. The longitudinal phase space before and after the X-band linearizer are shown in Figure 5, at a beam energy around $250 \mathrm{MeV}$ (before BC1).

The longitudinal phase space and beam current profile are shown in Figure 6 at the end of dogleg transport line, BC1 and $\mathrm{BC} 2$. A bunch compression ratio of 14 and 10 (for $5 \mathrm{kA}$ final beam current case) could be achieved in a first and second stage bunch compression, respectively. The final beam current can be pushed to above $5 \mathrm{kA}$. The higher peak current at two ends (double horn) is due to compression with larger energy correlation (chirp) from longitudinal wake fields in Linac2X. There is a similar effect in the LCLS case.

Electron beam properties at the end of Linac3X of this X-band driven FEL driver are shown in Figure 7 from ELEGANT simulation, including longitudinal phase space, longitudinally sliced energy spread, current profile and longitudinally sliced emittance. A similar plot is shown in Figure 8 for LCLS case. It is observed that the final beam current is over $5 \mathrm{kA}$ in this X-band driven FEL (3 kA for LCLS), and the sliced transverse emittance is similar with LCLS. 


\section{GENESIS simulation}

The electron bunch distribution generated from ELEGANT simulation is then fed into an undulator system, and the associated FEL performance is simulated and evaluated within the code GENESIS [9]. Same undulator setup is adopted for both this X-band driven FEL and the LCLS, which are described below. An ideal undulator is designed to have a short period of $\lambda_{w}=1.5 \mathrm{~cm}$, and the undulator strength $K$ is chosen to tune the resonant FEL wavelength being $\lambda_{r}=0.15 \mathrm{~nm}$, given the centroid energy of the electron bunch chosen at $14 \mathrm{GeV}$. No nonlinear magnetic field is included for the undulator model. No undulator tapering is adopted.

A comparison of the simulated FEL performance is shown in Figure 9 and Figure 10 below. As expected, this X-band driven FEL achieves a shorter FEL gain length, a higher saturation power and a higher FEL power at same undulator length (benefiting from a higher peak current of $5 \mathrm{kA}$ ), than the LCLS case.

\section{Acknowledgement}

The author would like to thank C. Adolphsen, P. Emma, T. Raubenheimer, J. Wu, M. Woodley, A. Chao, Z. Huang, Y. Ding, C. Limborg-Deprey for helpful discussions, C. Adolphsen for his support and guidance. The idea for this design is initially from C. Adolphsen et al. [1]. T. Raubenheimer proposed to push for higher peak current (in this paper $5 \mathrm{kA}$ ) by employing stronger X-band longitudinal wake fields in the last linac [2].

This work was supported by the DOE under Contract DE-AC02-76SF00515.

\section{References}

[1] C. Adolphsen et al., SLAC-PUB-14276 (2011).

[2] T. Raubenheimer, private communication (2011).

[3] P. Emma et al., Linac Coherent Light Source Conceptual Design Report, SLAC-R-593, SLAC (2002); P. Emma et al., "First lasing and operation of an angstrom-wavelength free-electron laser," Nature Photonics 4, 641-647 (2010).

[4] Yipeng Sun et al., "Beam Dynamics Study of X-Band Linac Driven X-Ray FELS," SLACPUB-14570 (2011); IPAC 2011, Spain, page 3128.

[5] Yipeng Sun, C. Adolphsen, C. Limborg-Deprey, T. Raubenheimer and J. Wu, "Low-charge, hard x-ray free electron laser driven with an X-band injector and accelerator," Phys. Rev. ST Accel. Beams 15, 030703 (2012).

[6] Yipeng Sun et al., "An X-band RF Based Hard X-ray FEL Design with Optics Linearization," to be submitted to Phys. Rev. ST Accel. Beams, SLAC-PUB-14511 (2012).

[7] K. Floettmann, ASTRA user manual, http://www.desy.de/mpyflo/Astradokumentation. 


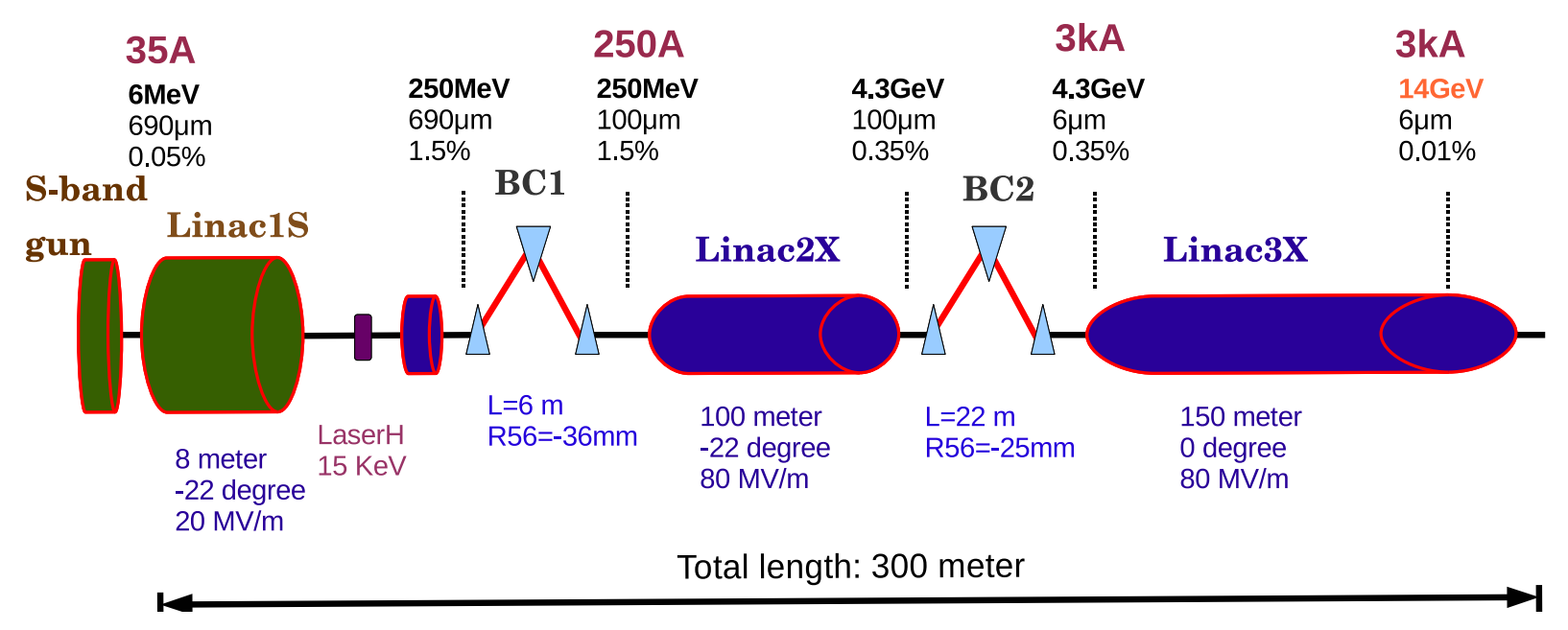

Figure 1: Sketch of a compact (300 m) hard X-ray FEL driver using X-band accelerators plus LCLS injector (up to BC1 end). An average 'real estate' acceleration gradient of $60 \mathrm{MV} / \mathrm{m}$ is assumed in the X-band accelerator structures. The linac for the hard X-ray FEL achieves a final electron bunch energy of $14 \mathrm{GeV}$ (same as LCLS case) with a peak current between $\mathbf{3} \mathbf{~ k A}$ and $\mathbf{5}$ kA (by tuning RF phase and then the bunch compression ratio). A final pulse duration length of 40-60 fs is achieved with a bunch charge of $250 \mathrm{pC}$. The sliced energy spread is between $0.01 \%-0.02 \%$, and the sliced normalized transverse emittance is below $0.6 \mu \mathrm{m}$.

[8] M. Borland, "elegant: A Flexible SDDS-Compliant Code for Accelerator Simulation," Advanced Photon Source LS-287, (September 2000).

[9] S. Reiche, "GENESIS 1.3: a fully 3D time-dependent FEL simulation code," Nucl. Instrum. Methods A 429, 243-248 (1999).

[10] H. Grote, F.C. Iselin, "The MAD Program (Methodical Accelerator Design) Version 8.15," CERN/SL/90-13 (AP) (1990). 

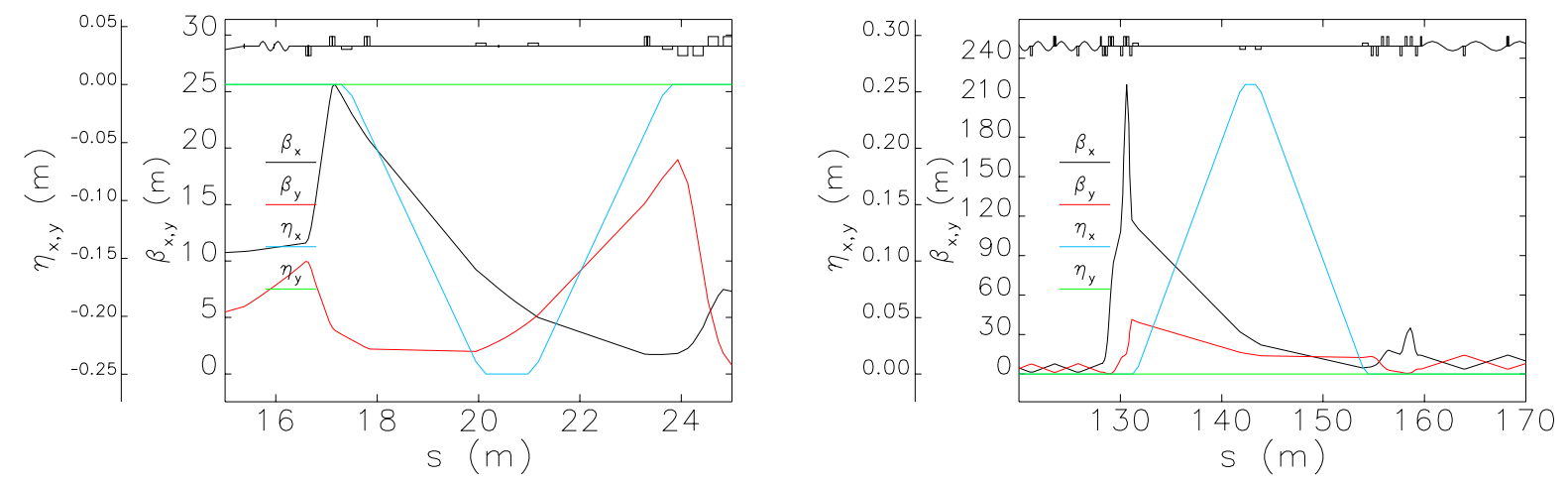

Figure 2: Beta functions and dispersion functions in the bunch compressors. Left: bunch compressor one which is exactly the same as LCLS BC1; Right: bunch compressor two which is similar with LCLS BC2. Black curve: horizontal beta function; red curve: vertical beta function; blue curve: horizontal dispersion function; green curve: vertical dispersion function. The transverse optics is quite similar with the LCLS case, which is designed to minimize the CSR induced transverse emittance growth. 


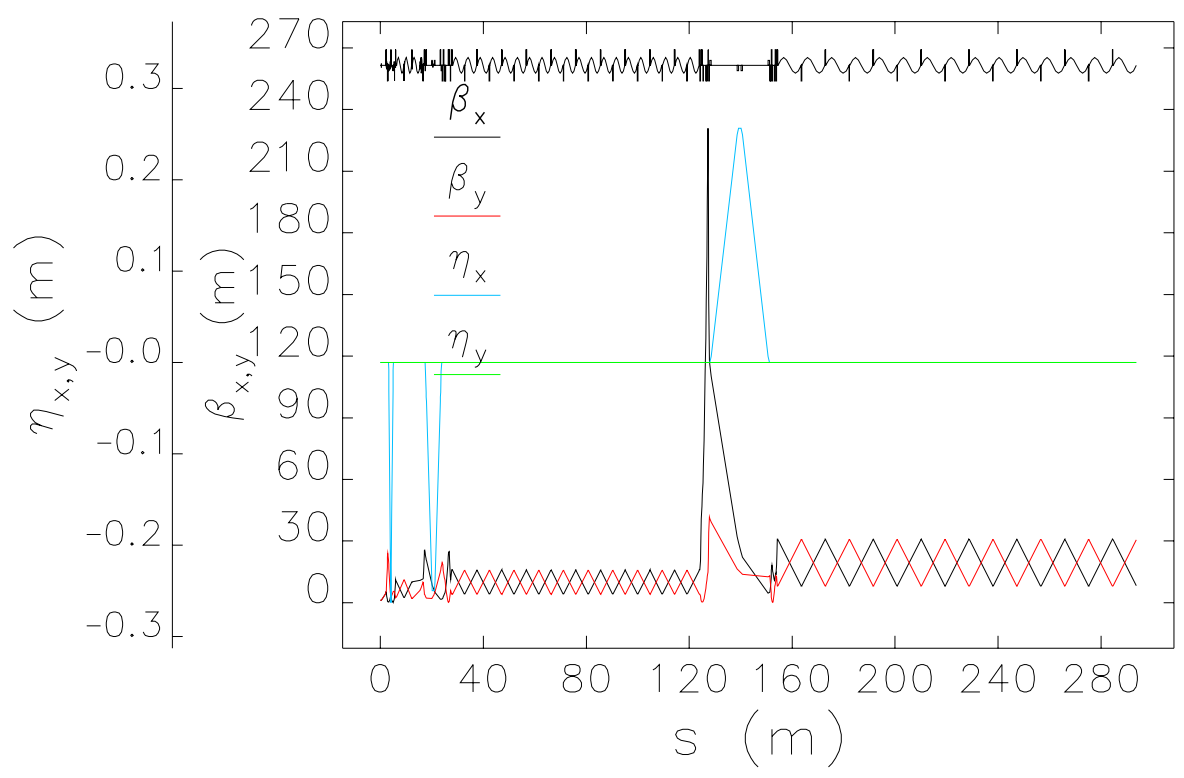

Figure 3: Beta functions and dispersion functions of the overall accelerator, from Linac1S (in Fig. 1) to Linac3X (in Fig. 1) end with a final beam energy of $14 \mathrm{GeV}$. Black curve: horizontal beta function; red curve: vertical beta function; blue curve: horizontal dispersion function; green curve: vertical dispersion function. Periodic FODO cells are employed for the X-band RF cavity based linac, with an average beta function of 10 meters and 20 meters in Linac2X and Linac3X, respectively. Simple matching sections are also employed between linac periodic cells and bunch compressors. The first stage bunch compressor is located at a place where the beam energy is 250 $\mathrm{MeV}$, while the second stage bunch compressor is at $4.3 \mathrm{GeV}$, just like the LCLS case. 

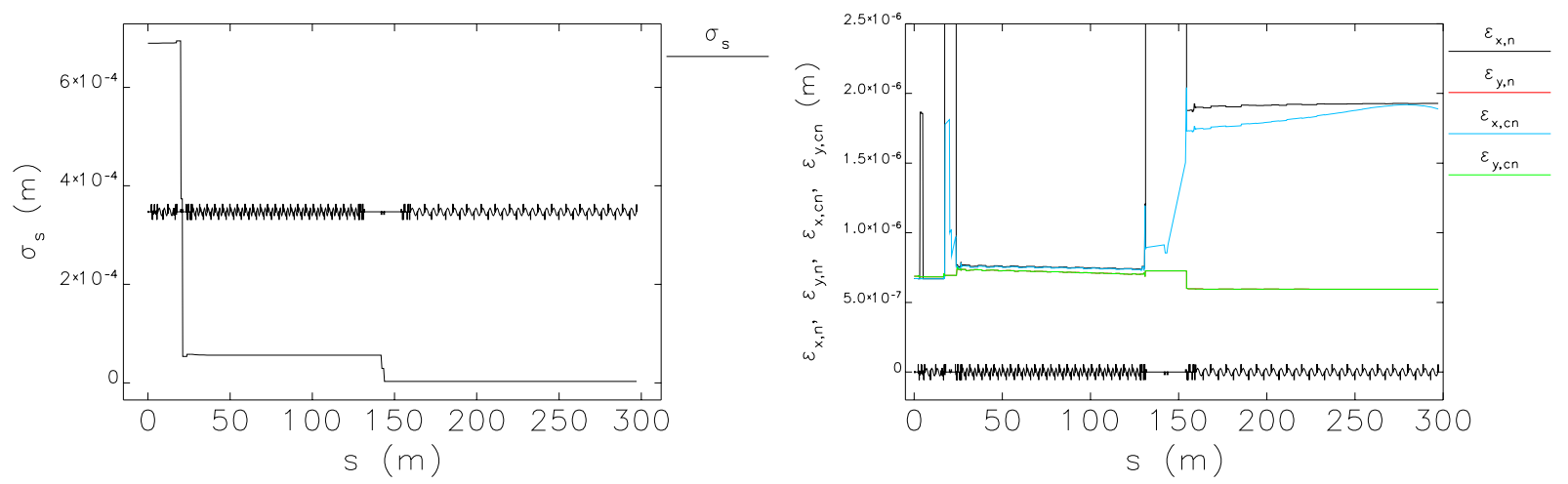

Figure 4: Left: RMS bunch length evolution along the linac, from ELEGANT simulation; Right: projected and linear dispersion subtracted emittance evolution along the linac, from ELEGANT simulation. A bunch compression ratio of 7 and 15 are achieved in a first and second stage bunch compressor (for $3 \mathrm{kA}$ final beam current case). The final RMS bunch length is around 6-7 $\mu \mathrm{m}$ with a peak current over $3 \mathrm{kA}$. In vertical plane the emittance is almost preserved. The horizontal emittance growth is mainly contributed from the coherent synchrotron radiation effects in the bunch compressors.
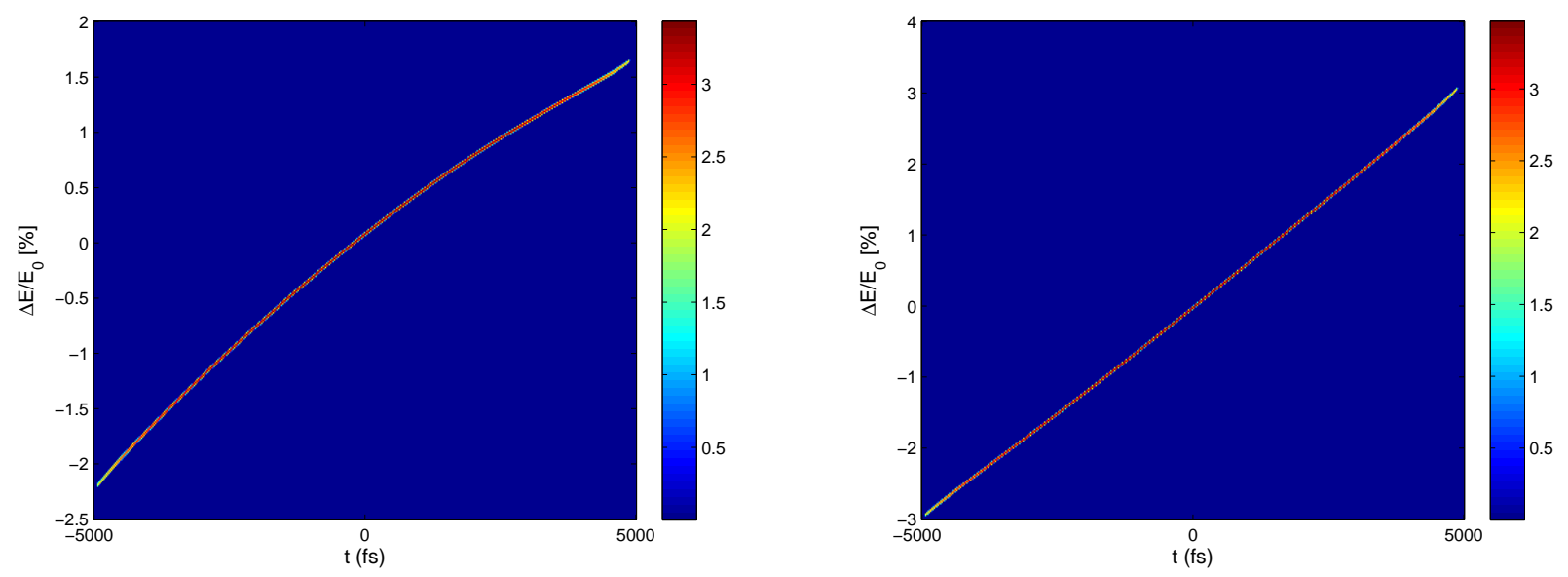

Figure 5: Longitudinal phase space before (left) and after (right) the X-band linearizer, at a beam energy around $250 \mathrm{MeV}$, from ELEGANT simulation. It is observed that the longitudinal phase space is a little bit over-linearized. The X-band linearizer RF phase is -160 degrees, and the oncrest voltage is $20 \mathrm{MV}$. 

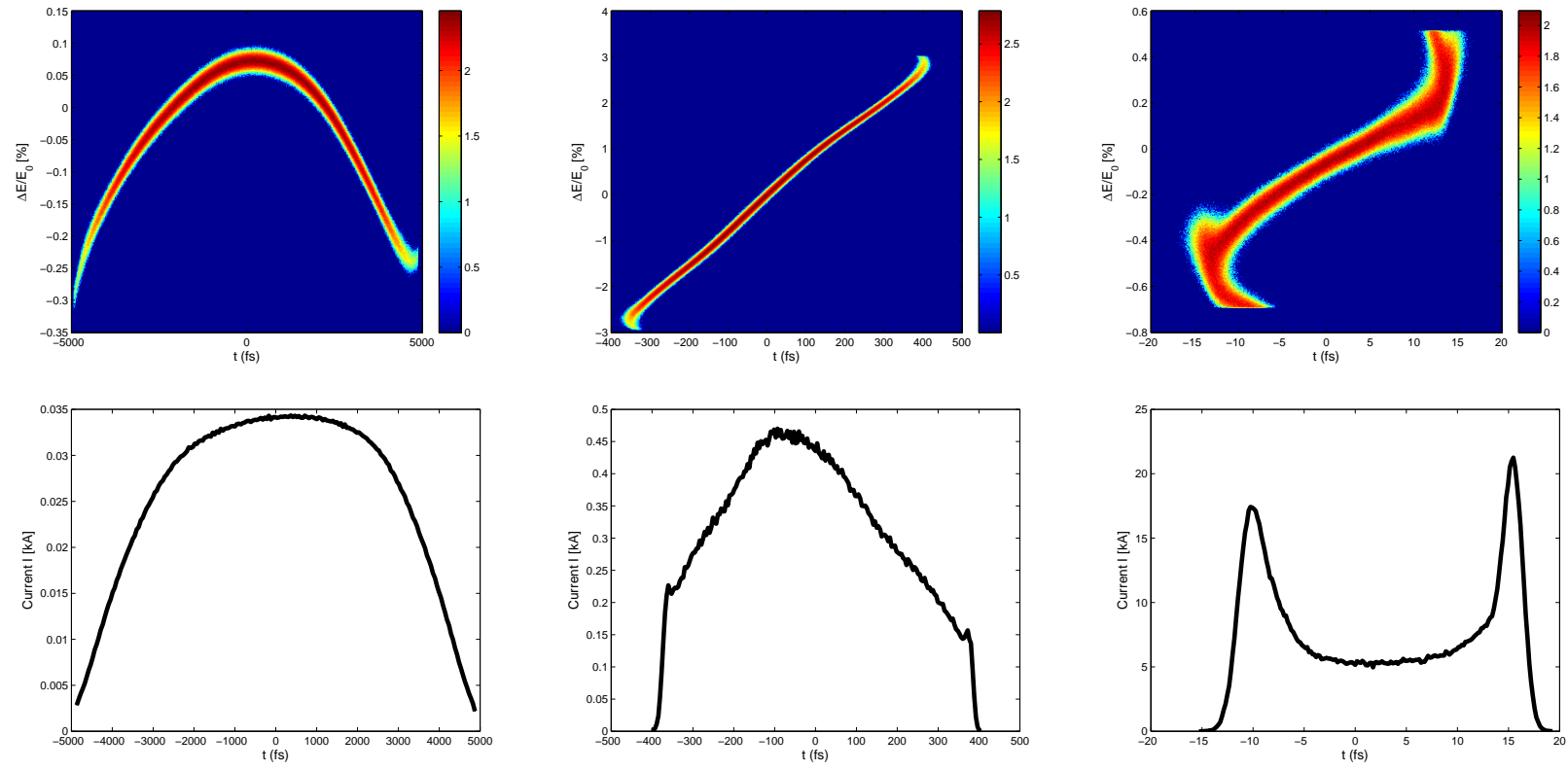

Figure 6: Longitudinal phase space (top) and peak current (bottom) evolution along the linac, from ELEGANT simulation. Left: at dogleg 1 end; Middle: at BC1 end; Right: at BC2 end. The configuration shown here is for a peak current over $5 \mathrm{kA}$. 

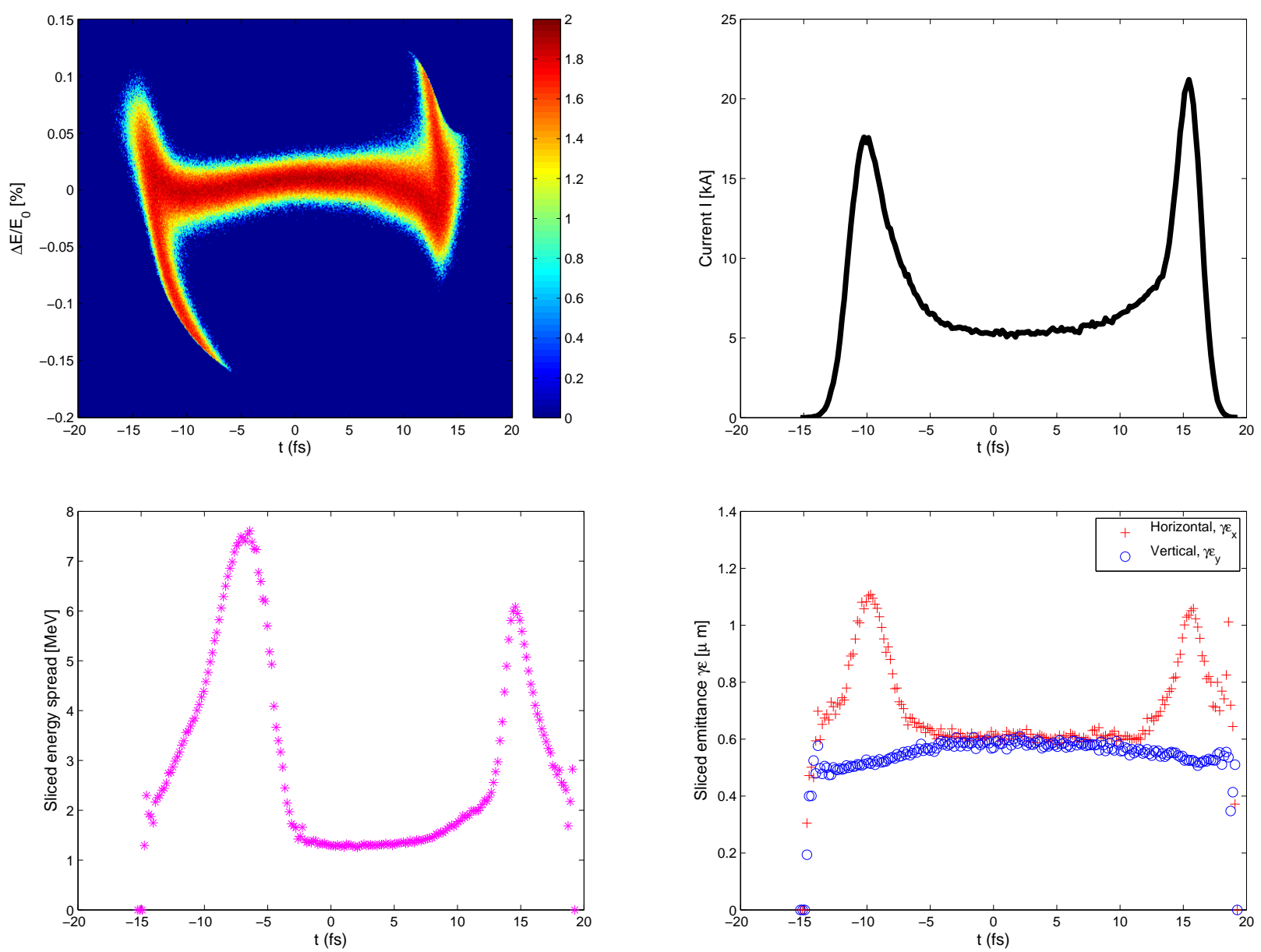

Figure 7: Electron beam properties at the end of Linac3X of this X-band driven FEL driver, from ELEGANT simulation. Left top: longitudinal phase space; left bottom: longitudinally sliced energy spread; right top: current profile; right bottom: longitudinally sliced emittance. A normalized transverse emittance of $0.6 \mu \mathrm{m}$ is preserved at the core part of the electron bunch, with a pulse duration length of $40 \mathrm{fs}$. A peak current of $5 \mathbf{~ k A}$ and a longitudinally sliced relative energy spread less than $2 \times 10^{-4}$ is achieved. The residual correlated energy offset established in Linac1 and Linac2X is removed in Linac3X with the help of strong X-band RF longitudinal wakefield, and a flat final energy profile is achieved at a final beam energy of $14 \mathrm{GeV}$. 

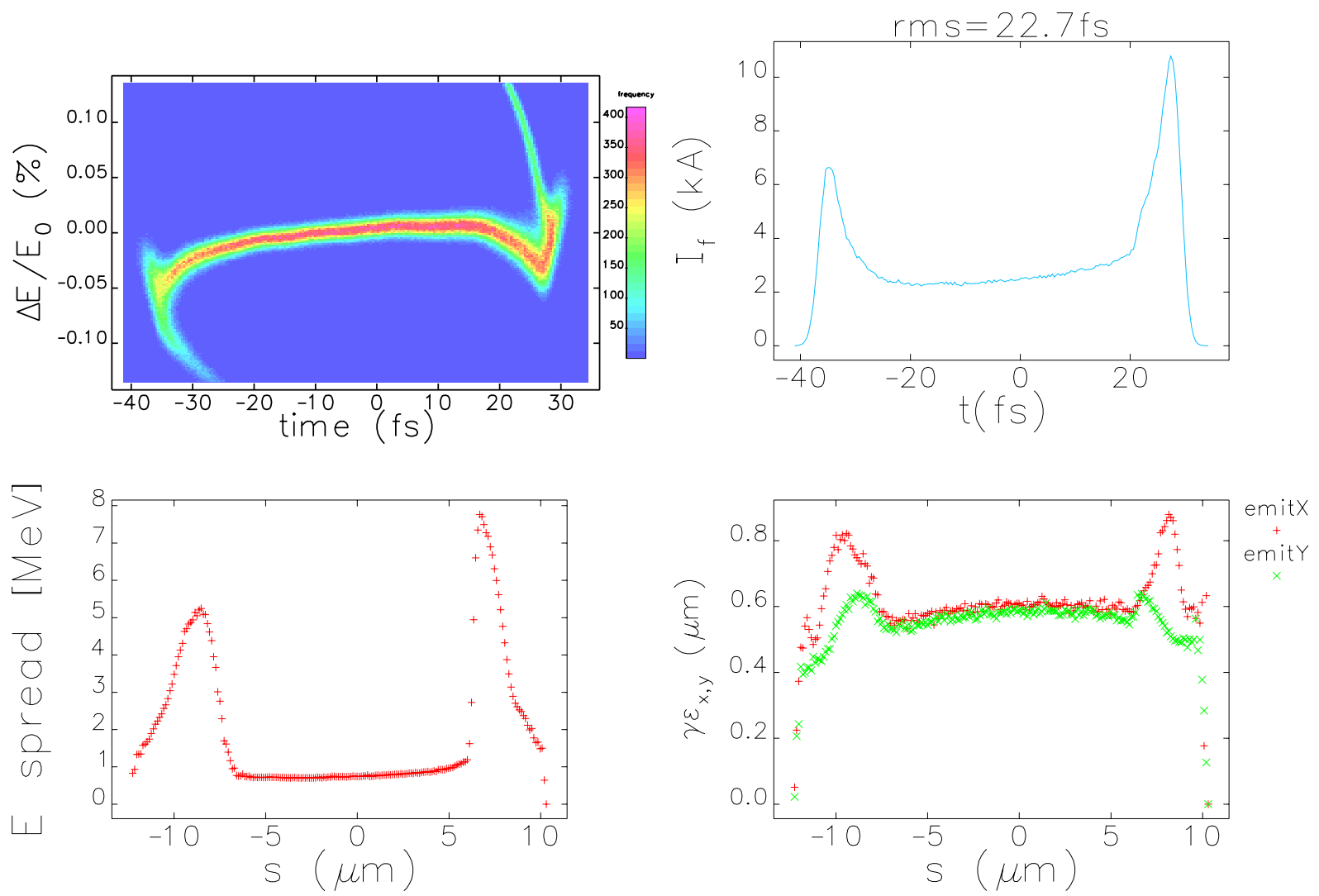

Figure 8: Electron beam properties at the end of LCLS Linac3, from ELEGANT simulation. Left top: longitudinal phase space; left bottom: longitudinally sliced energy spread; right top: current profile; right bottom: longitudinally sliced emittance. A normalized transverse emittance of 0.5-0.6 $\mu \mathrm{m}$ is preserved at the core part of the electron bunch, with a pulse duration length of $70 \mathrm{fs}$. A peak current of $3 \mathbf{~ k A}$ and a longitudinally sliced relative energy spread around $1 \times 10^{-4}$ is achieved. The residual correlated energy offset established in Linac1 and Linac2 is removed in Linac3 with the help of S-band RF longitudinal wakefield, and a flat final energy profile is achieved at a final beam energy of $14 \mathrm{GeV}$. 

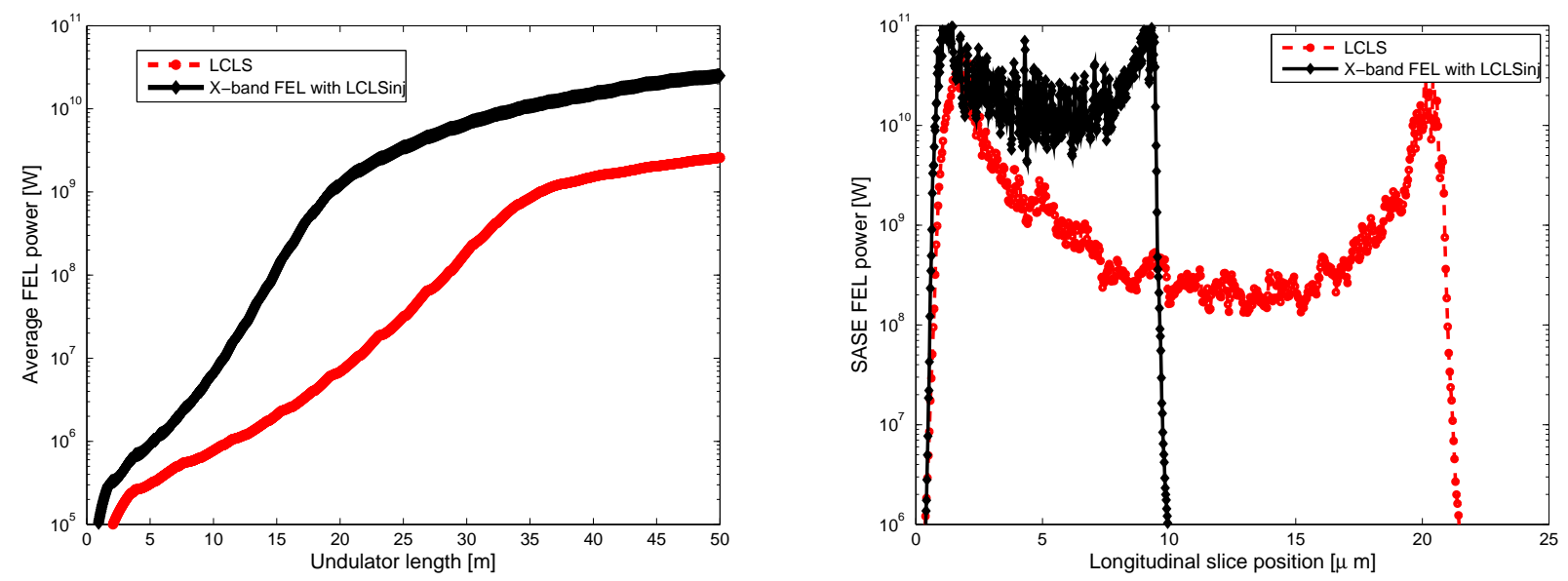

Figure 9: Left: FEL power evolution along the undulator; Right: FEL power temporal profile at $50 \mathrm{~m}$ into the undulator. Red: LCLS case with a final beam energy of $14 \mathrm{GeV}$; Black: the X-band driven FEL driver with LCLS injector (up to BC1 end) with a final beam energy of $14 \mathrm{GeV}$. The X-band driven FEL driver has a shorter FEL power gain length, and a higher sliced FEL power with same undulator length, due to its higher peak current of $5 \mathrm{kA}$. The simulation is performed in GENESIS with a dumped beam (1 million macro particles) from ELEGANT simulation at the linac end. The undulator configuration is the same for both the LCLS and the X-band driven FEL driver.

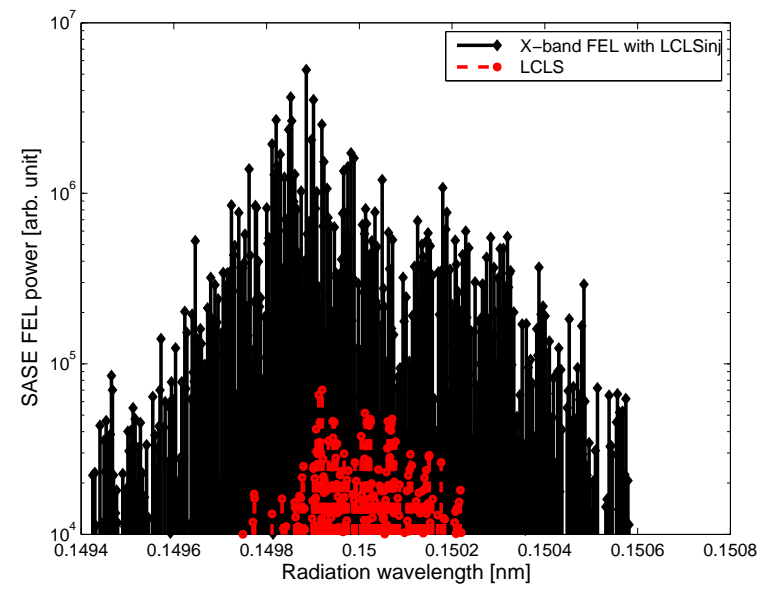

Figure 10: FEL power spectrum at $50 \mathrm{~m}$ into the undulator. Red: LCLS case with a final beam energy of $14 \mathrm{GeV}$; Black: the X-band driven FEL driver with LCLS injector (up to BC1 end) with a final beam energy of $14 \mathrm{GeV}$. The FEL radiation spectrum is wider for the X-band driven FEL driver as its sliced energy spread is larger, which is associated with its higher peak current of $5 \mathrm{kA}$. The simulation is performed in GENESIS with a dumped beam (1 million macro particles) from ELEGANT simulation at the linac end. The undulator configuration is the same for both the LCLS and the X-band driven FEL driver. 